\title{
Review on Anxiety: Its influence in Dental office, causes, recognition, prevention and management.
}

\author{
${ }^{1}$ Dr. Amol Bodhankar, ${ }^{2}$ Dr. Shailesh Pangaonkar, ${ }^{3}$ Dr. Uday Bodhankar, \\ ${ }^{4}$ Rupali Dongre. \\ ${ }^{I}$ (postgraduate student, Sharad Pawar dental College, Sawangi (meghe), Wardha. Bodhankar Residence, 46, \\ Malviya Nagar, Khamla, Nagpur - 440025, Maharashtra, India) \\ ${ }^{2}$ (Director, Central Institute of Behavioural sciences (CIBS), Srividya, 8, Navab layout, Tilak Nagar, Nagpur, \\ Maharashtra, India . \\ 3 (International President, Commonwealth association for health and disability(COMHAD),United Kingdom, \\ Nagpur, Maharashtra, India) \\ ${ }^{4}$ (Physiotherapist, Stroke Unit, Westmead Hospital, Sydney, Australia)
}

\begin{abstract}
Few people crave for popularity, few for power and few for money, but there is very very small category of people who simply focus on their work and rest everything follows them in abundance"

The aim of this review is to emphasize on Anxiety which is one of the common mental state in day to day to life due to traumatic events and stressful lifestyle, influence of others in our lives and absence of relaxation in schedule.

Anxiety has been neglected both by the operators and patients. However, many studies have proved association of Anxiety with increased pain during dental treatment, muscle contractions and altered state of concentration in everyday activities. This review includes aspect of anxiety, its causes, recognition, prevention and management.
\end{abstract}

Keywords: Anxiety, Dental treatment, Pain, Phobias, Relaxation.

\section{Introduction}

Anxiety has been shown to have marked influence on the pain threshold and healing of tissues, and thus it must be given importance.

Observing patient and their body language plays vital role in identification of mental state of the patient.

Increase in Stress may cause rise in cortisol levels and thus impairing body's immune functions and can make patients further more susceptible to infections.

The patient's levels of anxiety and preoperative pain have been shown to influence levels of postoperative dental Pain and anxiety is considered to be predictors of incomplete local anaesthesia. Highly fearful patients are more sensitive to pain in general and those who are dentally anxious are more sensitive to dental pain specifically. It has also been shown that more highly anxious patients report greater pain during dental procedures than normal controls (1)

A close relationship between pain and anxiety: the greater the anxiety, themore likely we are to interpret the sensation as pain, even the usual sensations could be interpreted as pain if the anxiety is high. (2) In a clinical study of children, it was found that anxiety is the strongest predictor of poor intra operative pain control. Similarly, during heightened anxiety, the pain threshold is lowered for patients (3)

\section{Anxiety and causes}

1) Panic Disorder: Panic disorder is an anxiety disorder characterized by recurring severe panic attacks. It may also include significant behavioral changes lasting at least a month and of ongoing worry about the implications or concern about having other attacks. The latter are called anticipatory attacks Panic disorder is not the same as agoraphobia (fear of public places), although many afflicted with panic disorder also suffer from agoraphobia. Panic attacks cannot be predicted, therefore an individual may become stressed, anxious or worried wondering when the next panic attack will occur

2) Obsessive Compulsive Disorder (OCD) : In this type of condition, patients are observed to repeat the activities Eg :Repeated washing of hands, etc

3) Post traumatic Stress disorder :This condition generally follows sudden physical or mental trauma such as accidents, death of close relatives which leads to sudden reduction of brain chemicals such as Serotonin. 
Review on Anxiety: Its influence in Dental office, causes, recognition, prevention and management.

\section{1) Social Anxiety disorder}

Social Phobia, also called Social Anxiety Disorder, is a condition whereby the individual feels overwhelming anxiety and excessive self-consciousness in everyday social situations. It's not that a person becomes afraid of people, but what other people may think of him or her. In a sense, the individual becomes extremely selfconscious in social environments. People with Social Phobia have a persistent, intense, and chronic fear of being watched and judged by others and being embarrassed or humiliated by their own actions. This fear may be so severe that it interferes with work, school, or any other ordinary social activity.

While many people with Social Phobia recognize that their fear of being around people is unusually excessive, they believe that they are unable to overcome it. And often, they worry for days or weeks prior to a social situation.Social Phobia can be limited to one type of situation, like speaking in public, or it can be as severe as to encompass many or all social situations.

Patients with social phobia are generally found to be apprehensive in meeting people, Dentists can identify this if the patient tries to avoid eye contact after entering into their clinic. (5)

5) Specific Phobias. Eg : Needle Phobias, or one of the common specific phobias are Arachnophobia or arachnephobia the fear of spiders and other arachnids such as scorpions.

\section{6)Generalized anxiety disorder.}

Serotonin is a chemical that helps maintain a "happy feeling," and seems to help keep our moods under control by helping with sleep, calming anxiety and bringing back serenity. Serotonin helps in regulation of mood, appetite, and sleep. Serotonin also has some cognitive functions, including memory and learning. Modulation of serotonin at synapses is thought to be a major action of several classes of pharmacological antidepressants.

\section{Anxiety manifests its impact on patients in many ways and its recognition can be based upon following}

facts :

Body (anxiety symptoms commonly associated with the body in general):

- Excessive sweating, feeling breathlessness, blurred vision

- Allergy problems, increase in allergies (number, sensitivity, reactions,Back pain, stiffness, tension, pressure, soreness, spasms, immobility in the back or back muscles, Burning skin sensation on the face, neck, ears, scalp, or shoulders

- $\quad$ craving for sugar and sweets

- Difficulty speaking, moving mouth, talking, co-ordination problems with the mouth or tongue

- Muscles that vibrate, jitter, tremor, or shake when used

- Impact on emotional state :

- A heightened fear of what people think of you, fear of losing identity among people, society.

- Afraid of being trapped in a place with no exits.

- Constant feeling of being overwhelmed.

- Fear of being in public and Fear of losing control

- Fear of making mistakes or making a fool of yourself to others

- Fears about irrational things, objects, circumstances, or situations

- Heightened self awareness, or self-consciousness

- Need to find nearest washrooms before you can feel comfortable

- Need to seat near exits.

- Feel like crying for no apparent reason.

Hearing/Ear(s) (anxiety symptoms commonly associated with hearing):

- Feel like there is something stuck in your ear, that your ear canal it plugged or blocked, that there is a pebble in your ear that you can't get out

- Low rumbling sounds

- Reduced hearing, frequent or intermittent reduced hearing or deafness in one or both ears

- Ringing in the ears, noises in the ears, noises in the head

- Pulsing in the ears, throbbing sound in the ear(s)

- Tickle or itch in your ear that you can't seem to get out.

- Clinical dental studies have indicated a strong relationship between endodontic postoperative pain and anxiety as well as intraoperative pain and anxiety (4) 


\section{Prevention}

Anxiety can be best prevented by following certain lifestyle,

Diet plays a vital role in prevention of anxiety, fruits such as Cherries, Almonds, Apples, and Yogurt (source of tryptophan), Walnuts are proved to aid in Serotonin secretion in brain and thus preventing Anxiety. Avoid foods high in saturated fats; consumption of fried foods, such as French fries, leads to sluggishness, slow thinking, and fatigue. Fats inhibit the synthesis of neurotransmitters by the brain in that they cause the blood cells to become sticky and to clump together, resulting in poor circulation, especially to the brain

Making life a relaxing experience must be the way life and duties should be performed, certain exercises such as Breathing, Meditation, Physical exercises have been proved to be very beneficial in preventing anxiety, other measure are :

1) Looking at early morning sunrise with eyes open for 2 minutes and if the sun gets brighter, look at it with closed eyes for 3 minutes.

2) Regular brisk walk in morning for atleast 20 minutes, and counting the steps while walking.

3) Evening time should be kept reserved for some sports or meditation, prayer, or any other spiritual activity.

4) Mindfulness towards our activities helps in the conservation of Serotonin hormone, which otherwise gets utilized in unproductive work.

Eg : Mindfulness while eating, mindfulness on the bolus, its chewing process, then the bolus getting mixed with saliva and going into the stomach. Avoid watching TV, playing video game, use of mobiles or any kind of multitasking during eating. This is an example of Mindfulness during eating, however any kind of Mindfulness aids in conservation of Serotonin and maintains the healthy mind set and thus maintain good concentration levels while working and aids in serving the society in a better way.

\section{Optimize Your Melatonin Levels :}

- According to Reiter RJ (May 1991), In humans, melatonin is produced by the pineal gland, a small endocrine gland located in the center of the brain but outside the blood-brain barrier. The melatonin signal forms part of the system that regulates the sleep-wake cycle by chemically causing drowsiness and lowering the body temperature, but it is the central nervous system that controls the daily cycle in most components of the endocrine systems

- Avoid watching TV or using your computer in the evening, at least an hour or so before going to bed. These devices emit blue light, which tricks your brain into thinking it's still daytime. Normally your brain starts secreting melatonin between 9 and $10 \mathrm{pm}$, and these devices emit light that may stifle that process.

- Make sure you get BRIGHT sun exposure regularly. Your pineal gland produces melatonin roughly in approximation to the contrast of bright sun exposure in the day and complete darkness at night. If you are in darkness all day long, it can't appreciate the difference and will not optimize your melatonin production.

- Sleep in complete darkness, or as close to it as possible. Even the slightest bit of light in your bedroom can disrupt your biological clock and your pineal gland's melatonin production.

- Take a hot bath 90 to 120 minutes before bedtime. This increases your core body temperature, and when you get out of the bath it abruptly drops, signalling your body that you are ready to sleep.

- Avoid using loud alarm clocks. Being jolted awake each morning can be very stressful. If you are regularly getting enough sleep, you might not even need an alarm.

- Be mindful of electromagnetic fields in your bedroom. EMFs can disrupt your pineal gland and its melatonin production, and may have other negative biological effects as well.. Move all electrical devices at least three feet away from your bed.

- Monday mornings have been found to be stressful as it is after the weekend, and people are found to be generally anxious. There is increased Cortisol production on Monday mornings, and thus optimize melatonin levels on Sunday evenings plays vital role in prevention of Anxiety.

\section{Management}

First and most important means for managing cases of anxiety, it is necessary that the clinician maintains his/her own serenity.

Generally it has been found that clinicians with heavy OPD are found to be stressed themselves, with the fear of losing their identity (for the reason, fear of improper handling of patients, fear of losing their image in society for the conditions where they are unable to treat patients due to lack of time or personal reasons) and it adds to anxiety among the clinician themselves. However Studies have shown that if the clinicians maintain the serenity, the surrounding environment also remains serene.

It depends upon how calmly the clinician tackles the case of anxiety, few patients in order to create their identity, may give Doctor their references about mutual friends which should be nicely entertained by the clinicians and if the patient is too much talkative, Patient must be brought to the area of interest in a polite way. 
Jacobson's progressive relaxation (and its shorter derivates) is probably the most widespread technique, especially in the USA; relaxation is obtained through the progressive contraction and relaxation of muscles.

An interesting study about the effects of relaxation on snake phobia was conducted by McGlynn et al. (1995) who divided 20 phobic patients in a relaxation group and a control group; after relaxation training, they were exposed to a phobic trigger, a snake in a cage, and patients were able to approach it; relaxation group patients showed a higher tolerance of distance to the snake and a lesser physiological arousal.

The use of relaxation training for hospitalised patients is often directed towards reducing surgery side effects such as pain. These techniques are often used in addition to anaesthetic drugs, or instead of anaesthetic drugs which sometimes cannot be prescribed (5)

Progressive muscle relaxation is a systematic technique for achieving a deep state of relaxation. It was developed by Dr. Edmund Jacobson more than fifty years ago. Dr. Jacobson discovered that a muscle could be relaxed by first tensing it for a few seconds and then releasing it. Tensing and releasing various muscle groups throughout the body produces a deep state of relaxation, which Dr. Jacobson found capable of relieving a variety of conditions, from high blood pressure to ulcerative colitis.(6)

The immediate effects of progressive muscle relaxation include all the benefits of the relaxation response described above. Long-term effects of regular practice of progressive muscle relaxation include:

- A decrease in generalized anxiety

- A decrease in anticipatory anxiety related to phobias

- Reduction in the frequency and duration of panic attacks

- Improved ability to face phobic situations through graded exposure

- Improved concentration

- An increased sense of control over moods

- Increased self-esteem

- Increased spontaneity and creativity (6)

Guidelines for Practicing Progressive Muscle Relaxation (or Any Form of Deep Relaxation) :

The following guidelines will help you make the most use of progressive muscle relaxation. They are also applicable to any form of deep relaxation you undertake to practice regularly, including guided visualization, and meditation.

1. Practice at least $\mathbf{2 0}$ minutes per day. Two 20-minute periods are preferable. Once a day is mandatory for obtaining generalization effects. (You may want to begin your practice with 30-minute periods. As you gain skill in relaxation technique, you will find that the amount of time you need to experience the relaxation response will decrease.)

2. Find a quiet location to practice where you won't be distracted. Don't permit the phone to ring while you're practicing. Use a fan or air conditioner to blot out

background noise if necessary.

3. Practice at regular times. On awakening, before retiring, or before meals are generally the best times. A consistent daily relaxation routine will increase the likelihood of generalization effects.

4. Practice on an empty stomach. Food digestion after meals will tend to disrupt deep relaxation.

5. Assume a comfortable position. Your entire body, including your head, should be supported. Lying down on a sofa or bed or sitting in a reclining chair are two ways of supporting your body most completely.

6. Loosen any tight clothing and take off shoes, watch, glasses, contact lenses, jewellery.

7. Make a decision not to worry about anything. Give yourself permission to put aside the concerns of the day. Allow taking care of yourself and having peace of mind to take precedence over any of your worries. (Success with relaxation depends on giving peace of mind high priority in your overall scheme of values.)

8. Assume a passive, detached attitude. This is probably the most important element.

You must adopt a "let it happen" attitude and be free of any worry about how well you are performing the technique. Do not try to relax. Do not try to control your body. Do not judge your performance. The point is to let go and experience whatever happens.

\section{Progressive Muscle Relaxation Technique}

Once you are comfortably supported in a quiet place, follow the detailed instructions below:

1.To begin, take three deep abdominal breaths, exhaling slowly each time. As you

exhale, imagine that tension throughout your body begins to flow away.

2. Clench your fists. Hold for 7-10 seconds and then release for 15-20

seconds. Use these same time intervals for all other muscle groups. 
Review on Anxiety: Its influence in Dental office, causes, recognition, prevention and management.

3. Tighten your biceps by drawing your forearms up toward your shoulders. Hold... and then relax.

Tense the muscles in your forehead by raising your eyebrows as far as you can.

Hold ... and then relax. Imagine your forehead muscles becoming smooth and

limp as they relax.

4. Tense the muscles around your eyes by clenching your eyelids tightly shut.

Hold... and then relax. Imagine sensations of deep relaxation spreading all

around them.

5. Tighten your jaws by opening your mouth so widely that you stretch the muscles around the hinges of your jaw. Hold ... and then relax. Let your lips part and allow your jaw to hang loose.

Tighten your buttocks by pulling them together. Hold ... and then relax. Imaginethe muscles in your hips going loose and limp.(6)

It has been concluded, that any type of relaxation technique takes mind to alpha phase of sleep, in which there is conversion of daily activities into feelings, Positive thoughts leads to positive emotions.

And hence it is advised to think positive, stress free before sleeping.

\section{Conclusion}

Anxiety is one of the most neglected aspects not just in dental office but overall, however it has been found to be common problem which leads to behavioural problems, difficulty to cope up with stressful situations or even dental and surgical procedure.

Thorough knowledge about anxiety and its management can lead to well being of human beings and will help us to spread serenity in our society, but this requires looking beyond the dental aspect and entering into the psyche of patients for better treatment outcomes.

\section{Acknowledgement}

We take this opportunity, to give heartfelt thanks to Dr.Shailesh Pangaonkar, for his constant guidance, due to his thorough understanding of psychological aspects of humans, and helping to remove mental and physical agony of people by serving through Central Institute of behavioural sciences (CIBS, Nagpur) Website : www.cibsindia.com

\section{References}

[1]. (Effectiveness of various medications on postoperative pain following complete instrumentation. J Endod 1994: 20: 345-354.)

[2]. Sokol D, Sokol S, Sokol C. A Review of non-intrusive therapies used to deal with anxiety and pain in the dental office. J Am Dent Assoc 1985: 110: 217-222.)

[3]. Gatchel RJ. Managing Anxiety and Pain during dental treatment. J Am Dent Assoc 1992: 123: 37-41.)

[4]. Nakai Y, Milgram P, Mancl L, Coldwell SE, Domoto PK, Ramsay DS. Effectiveness of local anesthesia in pediatric dental practice. J Am Dent Assoc 2000: 131: 1699- 1705.)

[5]. A brief literature review about relaxation therapy and anxiety, : Body, Movement and Dance in Psychotherapy: An International Journal for Theory, Research and Practice

[6]. Progressive Muscle Relaxation, Journal of Human Behavior in the Social Environment Volume 13, Issue 3, 2006. 